\title{
Transkriptionstabelle für die kyrillischen Zeichen der russischen Sprache
}

\begin{tabular}{|c|c|c|c|c|}
\hline \multicolumn{2}{|c|}{$\begin{array}{l}\text { Kyrillische } \\
\text { Buchstaben }\end{array}$} & \multicolumn{2}{|c|}{$\begin{array}{l}\text { Transkriblerte } \\
\text { Buchstaben }\end{array}$} & Bemerkungen \\
\hline A & a & $\mathbf{A}$ & $\mathbf{a}$ & \\
\hline Б & $\sigma$ & $\mathbf{B}$ & $\mathbf{b}$ & $\cdot$ \\
\hline B & B & $\mathrm{w}$ & w & \\
\hline$\Gamma$ & $\mathbf{r}$ & G & g & \\
\hline д & д & D & $\mathrm{d}$ & \\
\hline \multirow[t]{2}{*}{$\mathrm{E}, \ddot{\mathrm{E}}$} & e, è & & e & \\
\hline & & $J_{\theta}$ & jo & $\begin{array}{l}\text { am Anfang des Wortes, am Anfang der } \\
\text { Silbe nach Vokalen und nach den Zeichen } \\
\text { b und } b\end{array}$ \\
\hline Ж & ж & $\mathrm{Sh}$ & $\operatorname{sh}$ & . \\
\hline 3 & 8 & $\mathbf{s}$ & 8 & \\
\hline \multirow[t]{2}{*}{ и } & II & $\mathbf{I}$ & $\mathbf{i}$ & \\
\hline & 苗 & & $\mathbf{i}$ & unbezeichnet nach $\mathbf{n}$ und \\
\hline $\mathbf{K}$ & $\mathbf{K}$ & $\mathbf{K}$ & $\mathbf{k}$ & \\
\hline J & $\pi$ & $\mathbf{L}$ & 1 & \\
\hline $\mathbf{M}$ & $\mathbf{M}$ & $\mathbf{M}$ & $\mathrm{m}$ & \\
\hline $\mathbf{H}$ & H & $N$ & $\mathbf{n}$ & \\
\hline 0 & 0 & 0 & 0 & \\
\hline$\Pi$ & II & $P$ & $p$ & \\
\hline $\mathbf{P}$ & p & $\mathbf{R}$ & $\mathbf{r}$ & \\
\hline \multirow[t]{2}{*}{$\mathrm{C}$} & $\mathrm{c}$ & $\mathrm{Sg}$ & ss & \\
\hline & & $\mathbf{s}$ & s & $\begin{array}{l}\text { am Anfang des Wortes vor einem Konso- } \\
\text { nanten und zwischen zwei Konsonanten }\end{array}$ \\
\hline $\mathrm{T}$ & $\mathbf{T}$ & $\mathrm{T}$ & $\mathbf{t}$ & \\
\hline $\mathrm{y}$ & $y$ & $\mathbf{U}$ & $\mathbf{u}$ & \\
\hline$\Phi$ & $\Phi$ & $F$ & f & \\
\hline $\mathbf{x}$ & $\mathbf{x}$ & $\mathrm{Ch}$ & $\mathrm{ch}$ & \\
\hline \multirow[t]{2}{*}{ ц } & ц & $\mathbf{z}$ & $\mathbf{z}$ & \\
\hline & & & $\mathrm{tz}$ & nach Vokal \\
\hline ч & $\mathbf{4}$ & Tsch & tsch & \\
\hline III & III & Sch & sch & \\
\hline \multirow[t]{4}{*}{ III } & m & Schtsch & schtsch & \\
\hline & $\mathbf{b}$ & & - & bleibt unbezeichnet, siehe unter e \\
\hline & ы & & $\mathbf{y}$ & \\
\hline & $\mathbf{b}$ & & - & bleibt unbezeichnet, siehe unter e \\
\hline$\vartheta$ & a & $\mathbf{E}$ & e & \\
\hline ю & 10 & $\mathrm{Ju}$ & ju & \\
\hline я & я & $\mathrm{Ja}$ & ja & \\
\hline
\end{tabular}

